\title{
Electroacupuncture to treat gastroesophageal reflux disease: study protocol for a randomized controlled trial
}

\author{
Gajin $\operatorname{Han}^{1,2}$, Jungtae Leem ${ }^{3}$, Hojung Lee ${ }^{3}$ and Junhee Lee ${ }^{1,4^{*}}$
}

\begin{abstract}
Background: Gastroesophageal reflux disease lowers the quality of life and increases medical costs. Electroacupuncture has been used to ease symptoms and improve gastrointestinal motility in patients with gastroesophageal reflux disease. The main purposes of this study are to evaluate the efficacy and safety of this procedure.

Methods/design: This is a protocol for a randomized, patient-blinded, assessor-blinded, sham-controlled trial. Sixty participants with symptoms of gastroesophageal reflux disease, who have previously undergone standard treatment, will be recruited from August 2015 at Kyung Hee University Korean Medicine Hospital. The participants will be allocated to either the electroacupuncture $(n=30)$ or the sham electroacupuncture group $(n=30)$; the allocation will be concealed from both the participants and the assessors. The EA group will undergo penetrating acupuncture at 18 fixed points and two optional points chosen using the pattern identification for gastroesophageal reflux disease. Electrical stimulation will be applied at some of the acupoints. The sham electroacupuncture group will undergo nonpenetrating acupuncture without electrical stimulation at 18 nonspecific points, each of which will be only $2 \mathrm{~cm}$ away from the true acupoints used in the electroacupuncture group. In both groups, the procedure will be performed using the Park device. The treatment will last for 6 weeks (with two sessions each week), and the outcome will be evaluated at baseline, 3 weeks, and 6 weeks. The primary outcome will be the proportion of responders with adequate symptom relief, whereas the secondary outcomes will comprise the results of the Nepean dyspepsia index; the Korean gastrointestinal symptom rating scale; the EQ-5D'm; levels of gastrin, motilin, and inflammatory cytokines; the perceived stress scale; the qi-stagnation questionnaire; the patient global impression of change; and the spleen qi deficiency questionnaire.
\end{abstract}

Discussion: The results of this trial will provide information about the efficacy and safety of electroacupuncture in the treatment of gastroesophageal reflux disease symptoms, as well as evidence regarding the use of electroacupuncture to treat gastroesophageal reflux disease in real clinical practice.

Trial registration: Clinical Research Information Service Identifier, KCT0001653. Registered on 12 October 2015.

Keywords: Gastroesophageal reflux disease, Electroacupuncture, Park device, Proportion of responders, Pattern identification

\footnotetext{
*Correspondence: ssljh@khu.ac.kr

'Korean Medicine Clinical Trial Center, Kyung Hee University Korean Medicine Hospital, 23 Kyungheedae-ro, Dongdaemun-gu, Seoul 02447, Republic of Korea

${ }^{4}$ Department of Sasang Constitutional Medicine, College of Korean Medicine, Kyung Hee University, 26 Kyungheedae-ro, Dongdaemun-gu, Seoul 02447, Republic of Korea

Full list of author information is available at the end of the article
} 


\section{Background}

Recently, the prevalence of gastroesophageal reflux disease (GERD) in Asia has been increasing, and GERD has now become an important disease [1-3]. Most patients with GERD require long-term therapy, as it is a chronic condition [4]. The standard treatment for GERD is known as a proton pump inhibitor (PPI), which heals the esophageal mucosa and eases the symptoms of GERD; the PPI has become a mainstay of GERD treatment [5]. However, approximately $20 \%$ to $30 \%$ of GERD patients continue to experience symptoms despite PPI treatment [6].

For this reason, interest is growing in complementary and alternative medicine-including acupuncture [5]. Recently, acupuncture has been reported to possibly ease gastrointestinal symptoms [7] and improve esophageal motility [8]. For instance, according to several previous animal studies [9-11], electroacupuncture (EA) increases abnormally low esophageal sphincter pressure (ESP) and reduces the frequency of transient lower esophageal reflux relaxations (TLESRs); in so doing, the treatment increases esophageal motility. GERD usually develops because of a decrease in ESP and the esophageal clearance capacity, as well as an increase in TLESR frequency [12]. Taken together, EA likely ameliorates GERD by controlling esophageal motility. However, little has been reported regarding the efficacy of EA in GERD; to our knowledge, no randomized controlled trials (RCTs), where a control group undergoes a sham procedure, have been carried out to evaluate the effect of EA on GERD in humans.

On another note, both gastrin and motilin are related to ESP, yet the influence of EA on the levels of these proteins has been disputed in previous studies $[11,13-16]$. Therefore, with a view to resolving this controversy, we will evaluate the differences in gastrin and motilin levels after EA. Meanwhile, one report has stated that inflammatory mediators are associated with esophageal motility in patients with GERD [17]. For this reason, before and after treatment, we will test for several inflammatory cytokines present in GERD to evaluate the influence of EA on the levels of these cytokines.

In clinical practice, many patients who continue to suffer symptoms of GERD despite standard treatments turn to traditional Korean medicine-specifically EA. Nonetheless, there remains insufficient evidence from well-designed RCTs to support EA use. Hence, we have designed this trial to evaluate the efficacy and safety of partially individualized EA in patients with GERD who have already undergone standard therapy (PPIs or lifestyle modification). In the trial, an EA group will be compared with a sham EA control group. In addition, we will clarify the influence of EA on gastrointestinal (GI) hormones and inflammatory cytokines in patients with GERD.

\section{Methods/design}

This protocol was prepared according to the Standard Protocol Items: Recommendations for Interventional Trials (SPIRIT) 2013 statement. See Additional file 1 for the completed SPIRIT checklist.

\section{Hypothesis \\ Partially individualized EA treatment is superior to sham EA in terms of easing the symptoms of GERD.}

\section{Design}

A single-center, parallel, randomized, patient-blinded, assessor-blinded, sham EA-controlled trial will be conducted at the Kyung Hee Korean Medicine Clinical Trial Center, which is affiliated with Kyung Hee University Korean Medicine Hospital in Seoul, Republic of Korea, from August 2015 to January 2016. Figure 1 shows the planned timeline of the trial.

\section{Inclusion and exclusion criteria}

Participants will (1) be ages 19 to 75 years; (2) have been diagnosed with GERD (either erosive or nonerosive) no more than a year before the trial begins; (3) have had one or more of certain GERD symptoms in the month before the trial begins (heartburn, regurgitation, dyspepsia, epigastric pain, belching, nausea, globus (feeling of a lump in the esophagus), dysphagia, coughing, or hoarseness [18]); (4) have had these symptoms on more than 4 days (in the case of mild symptoms) or more than 2 days (in the case of moderate and severe symptoms) during the 7 days before the trial begins; (5) have continued to experience the symptoms stated above despite standard treatment for GERD-using either medication or lifestyle modification, or both-for 4 weeks during the year before the trial begins; and (6) agree to participate in this study, voluntarily signing a written informed consent form. Symptoms that are easily tolerated, with minimal discomfort not affecting normal activities, are defined as "mild"; those that are sufficient to affect normal activities are classified as "moderate," and severe symptoms comprise those that markedly affect normal activities [19].

Participants will be excluded if they (1) have any current symptoms related to a structural disease that has been confirmed by endoscopy (e.g., gastrointestinal cancer, eosinophilic esophagitis, candida esophagitis, etc.); (2) have severe dysphagia, hematemesis, weight loss, or hematochezia; (3) have suffered from gastrointestinal cancer during the 5 years before the study begins; (4) have received chemotherapy for cancer during the month before the study begins; (5) have been diagnosed with ischemic heart disease (e.g., angina pectoris or myocardial infarction); (6) have an artificial cardiac pacemaker in the chest; (7) have experienced a hypersensitivity reaction after an acupuncture treatment, or show any other contraindications; (8) are taking part in any 


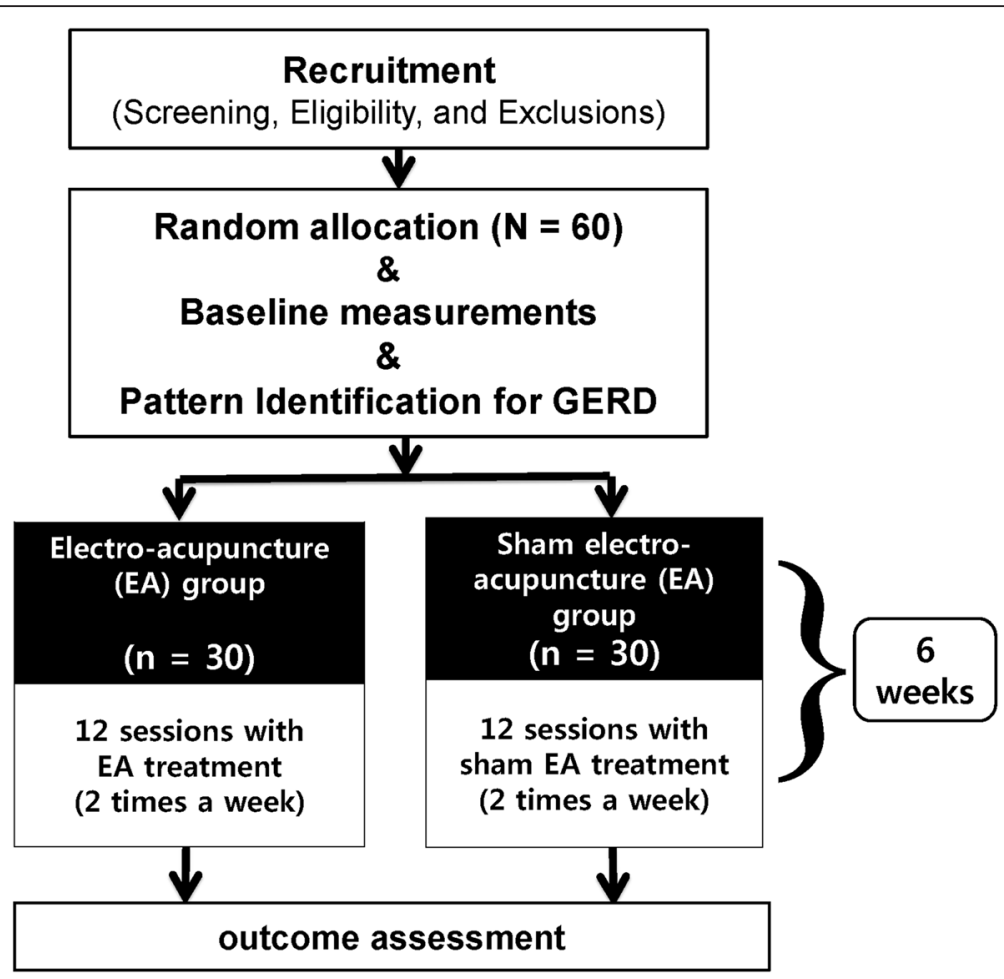

Fig. 1 Timeline of the study

other clinical trial that could affect the results of this trial, or are being treated for GERD using any other Korean medical method (a minimum wash-out period of 2 weeks will be required for participation in this trial); (9) have difficulties attending the trial (e.g., serious mental illness, dementia, drug addiction, severe disorders in vision or hearing, illiteracy, time constraints, etc.), and (10) are pregnant or breastfeeding.

\section{Recruitment}

We will advertise the study on the notice boards and the homepage of the hospital, as well as in local newspapers, and the investigators who are Korean medical doctors, will obtain informed consent from all participants. Specifically, two written informed consent forms (a general consent form and an additional consent provision for the collection and use of the participants' biological material) will be given to the participants. After the participants sign the forms voluntarily, the participants will go through the screening test. We will provide a copy of the consent form and other related documentation to the participants upon request.

\section{Randomization and allocation concealment}

Sixty participants will be randomly assigned to either the EA or sham EA group. A random number table will be generated using block randomization with blocks of four by $\mathrm{R}$ for windows 3.2.1 ( $\mathrm{R}$ Foundation for Statistical Computing, Vienna, Austria); to this end, the block randomization function will be used with blocks of four. The allocations will be kept sealed in a double-locked cabinet by an independent statistician. If the participants pass the screening test, they will be allocated according to the procedure above to conceal the allocation from both investigators and participants. After allocation, the statistician will email a unique random number and participant group allocation to both the clinical research coordinator and the Korean medicine doctors (KMDs). This feedback document will be kept in the master file for the trial.

\section{Blinding}

In this study, the participants, the outcome assessors, and the data managers, including the statistician, will be blinded to the group allocation. The acupuncture practitioner will not be blinded because of the characteristics of the Park sham device. The investigators will not give any clues to the participants regarding group allocation, nor will the participants from one group meet those from the other group. To evaluate the success of blinding, a blinding index will be assessed after the participants' final treatment. Blinding will be maintained until the study is finished. However, if a serious adverse event (AE) occurs, blinding will be lifted, and investigators will notify the participant involved of his/her group allocation in accordance with the standard operating procedures (SOPs). 


\section{Intervention}

In Table 1, we have provided detailed information regarding the EA treatment to be used; the treatment will be based on the revised Standards for Reporting Interventions in Clinical Trials of Acupuncture (STRICTA) [20]. Furthermore, we have tried to reconstruct a real clinical environment in the study design, using partially individualized EA treatments based on both meridian theory and the consensus of Korean medicine gastroenterologists. Individualized treatment will be carried out according to the participant's pattern identification. At the first visit, the participant will respond to a GERD pattern identification tool [21], and the pattern of the participant will be identified as one of following four types: "liver qi invading the stomach," "spleenstomach weakness," "spleen-stomach dampness-heat," and "stomach yin deficiency." The participants' identified types will not change for the duration of the study.

The treatment will consist of 12 sessions across 6 weeks (two sessions each week); it will be performed by KMDs with more than 5 years of clinical experience in acupuncture, who will go through training and simulation to ensure identical acupuncture treatment in accordance with the SOPs. The minimum interval between treatment sessions will be 2 days. Lifestyle will be modified in both groups.

\section{EA group}

The number of inserted needles per participant in one session will range from 18 to 20 . The basic points and optional points used will be included in the acupuncture treatment regimen. All participants will undergo acupuncture treatment at 18 fixed points. The details of the acupoints are shown in Table 1. Of these points, electrical stimulation will only be applied to ST36, a point $0.5 \mathrm{~cm}$ below ST36, PC6, LI4 (bilateral), CV10, and CV13 (unilateral). In addition, optional points will be selected on the basis of the participant's pattern identification; the details are shown in Table 1.

We will use a Park sham device (Acuprime, Exeter, UK) with real penetrating needles [22]; the inserted needle of this device is a sterilized stainless steel needle (DongBang Acupucture Inc., Bundang, Sungnam, Republic of Korea) $40 \mathrm{~mm}$ in length and $0.25 \mathrm{~mm}$ in diameter. The needles will be inserted to a depth of between $5 \mathrm{~mm}$ and $30 \mathrm{~mm}$, and they will remain in place for 20 minutes. In the case of EA points, the needles will be stimulated using a mixed electric current at a frequency of $2 / 6 \mathrm{~Hz}$; to this end, the "fast plus slow" mode of the ES-160 (ITO Co., Ltd., Tokyo, Japan) will be used. The stimulation intensity will be adjusted depending on the degree of the participant's tolerance.

\section{Sham EA group}

Participants in the sham EA group will be treated using 18 fixed acupoints. However, acupoints based on individual pattern identification will not be used. We will use a Park sham device with sham (blunted) needles [22] that do not penetrate the skin; this device has been described in previous studies [23-25]. At the EA points, the same accessories of the EA equipment will be assembled, and there will be a timer sound and a blinking light; however, no electrical current will be applied.

\section{Lifestyle modification}

In accordance with the clinical practice guidelines for GERD [26], as well as the SOP, lifestyle modification will be taught to participants by the KMD at every visit. Lifestyle modification will comprise (1) avoidance of foods that worsen GERD symptoms (e.g., coffee, alcohol, chocolate, fat, etc.), (2) avoidance of foods that cause heartburn (e.g., spicy food, fruit, carbonated drinks, etc.), (3) changes to lifestyle that decrease the exposure of the lower esophagus to gastric acid (e.g., weight loss, cessation of smoking and of drinking alcohol, raising the upper part of the bed, going to bed 2-3 hours after a meal) [27].

\section{Distribution of rescue medicine}

With regard to ethics, we will distribute the rescue medicine, named Norumo Dual Action Suspension (Il-Yang Pharmaceutical Co., Ltd., Gangnam, Seoul, Republic of Korea), to all participants. If participants experience intolerable GERD symptoms between visits, they will be permitted to take one pack. The ingredients of the medicine are sodium alginate, sodium bicarbonate, and calcium carbonate. The number of rescue medicines administered will be evaluated at each visit. If the number between visits is more than three, then the primary outcome, adequate relief (AR), will be classified as "no relief," regardless of the participant's answer.

\section{Withdrawal criteria}

Participants will be withdrawn from the study if they (1) miss a scheduled EA treatment three times in a row or (2) take forbidden medication, herbal medicine, or undergo surgery related to GERD during the study period.

\section{Drugs forbidden by the study protocol}

The following classes of drugs, all of which are used to treat symptoms of GERD, will not be allowed: PPIs, histamine receptor antagonists, prokinetics, mucosal protective drugs, antacids, anti-depressants, antianxiety drugs, lower esophageal sphincter agents, and herbal medicine.

\section{Outcome measurement}

An independent assessor will give the participants a set of outcome measures; a schedule of these measures is summarized in Table 2. 
Table 1 Acupuncture treatment details based on the STRICTA 2010 checklist

\begin{tabular}{|c|c|}
\hline Item & Detail \\
\hline \multirow[t]{3}{*}{$\begin{array}{l}\text { 1. Acupuncture } \\
\text { rationale }\end{array}$} & $\begin{array}{l}\text { 1a) Style of acupuncture } \\
\text { - EA based on traditional meridian theory. }\end{array}$ \\
\hline & $\begin{array}{l}\text { 1b) Reasoning for treatment provided, } \\
\text { based on historical context, } \\
\text { literature sources, and/or consensus methods, } \\
\text { with references where appropriate } \\
\text { - Partially individualized manual EA treatments } \\
\text { based on the traditional meridian theory, } \\
\text { literature review, consensus by the experts in } \\
\text { GERD and acupuncture, and clinical experience } \\
\text { of Korean medicine gastroenterologist. }\end{array}$ \\
\hline & $\begin{array}{l}\text { 1c) Extent to which treatment was varied } \\
\text { - Partially individualized EA treatment, i.e., } \\
\text { fixed points plus optional points according } \\
\text { to pattern identification of the participant. }\end{array}$ \\
\hline \multirow[t]{7}{*}{$\begin{array}{l}\text { 2. Details of } \\
\text { needling }\end{array}$} & $\begin{array}{l}\text { 2a) Number of needle insertions per subject } \\
\text { per session (mean and range where relevant) } \\
\text { - From } 18 \text { to } 20 \text {. }\end{array}$ \\
\hline & $\begin{array}{l}\text { 2b) Names (or location if no standard name) } \\
\text { of points used (uni/bilateral) } \\
\text { - Eighteen fixed points: ST36, a point } 0.5 \text { cun below } \\
\text { ST36, PC6, LI4, LR3, SP4, SP9, GB34 (bilateral), CV10, } \\
\text { and CV13 (unilateral). } \\
\text { - Optional points according to individual pattern } \\
\text { identification } \\
\text { (1) "Liver qi invading the stomach" type: } \\
\text { SP11, LR2 (Rt. side) } \\
\text { (2) "Spleen-stomach weakness" type: CV12 (one point), } \\
\text { ST41 (Rt. side) } \\
\text { (3) "Spleen-stomach dampness-heat" type: LR13, SP3 } \\
\text { (Rt. side) } \\
\text { (4) "Stomach yin deficiency" type: CV12 (one point), } \\
\text { ST44 (Rt. side) }\end{array}$ \\
\hline & $\begin{array}{l}\text { 2c) Depth of insertion, based on a specified unit } \\
\text { of measurement, or on a particular tissue level } \\
\text { - From } 5 \text { to } 30 \mathrm{~mm} \text {. }\end{array}$ \\
\hline & $\begin{array}{l}\text { 2d) Response sought } \\
\text { (e.g., de qi or muscle twitch response) } \\
\text { - none }\end{array}$ \\
\hline & $\begin{array}{l}\text { 2e) Needle stimulation (e.g., manual, electrical) } \\
\text { - Electrical stimulation: } 2 / 6 \mathrm{~Hz} \text { mixed } \\
\text { (ITO ES-160, Japan) } \\
\text { - Acupoints with electrical stimulation: ST36, } \\
\text { a point } 0.5 \text { cun below ST36, PC6, LI4 (bilateral), } \\
\text { CV10, and CV13 (unilateral). }\end{array}$ \\
\hline & $\begin{array}{l}\text { 2f) Needle retention time } \\
\text { - } 20 \text { minutes. }\end{array}$ \\
\hline & $\begin{array}{l}\text { 2g) Needle type } \\
\text { (diameter, length, and manufacturer or material) } \\
\text { - Park sham device with real and sham needle, i.e., } \\
\text { sterilized stainless steel needle } \\
\text { (0.25 } \times 40 \mathrm{~mm} \text {, Dongbang Acupuncture Inc., } \\
\text { Bundang, Sungnam, Korea). }\end{array}$ \\
\hline \multirow[t]{2}{*}{$\begin{array}{l}\text { 3. Treatment } \\
\text { regimen }\end{array}$} & $\begin{array}{l}\text { 3a) Number of treatment sessions } \\
\text { - Twelve treatment sessions in both EA and } \\
\text { sham EA groups. }\end{array}$ \\
\hline & $\begin{array}{l}\text { 3b) Frequency and duration of treatment sessions } \\
\text { - Twice weekly for } 6 \text { weeks, } 20 \text { minutes for each } \\
\text { session. }\end{array}$ \\
\hline
\end{tabular}

Table 1 Acupuncture treatment details based on the STRICTA 2010 checklist (Continued)

\begin{tabular}{|c|c|}
\hline $\begin{array}{l}\text { 4. Other } \\
\text { components } \\
\text { of treatment }\end{array}$ & $\begin{array}{l}\text { 4a) Details of other interventions administered } \\
\text { to the acupuncture group (e.g., moxibustion, } \\
\text { cupping, herbs, exercises, lifestyle advice) } \\
\text { - Except for lifestyle modification and rescue medicine, } \\
\text { other interventions during the study period will not } \\
\text { be allowed. } \\
\text { 4b) Setting and context of treatment, including } \\
\text { instructions to practitioners, and information } \\
\text { and explanations to patients } \\
\text { - Korean Medicine Clinical Trial Center in } \\
\text { University hospitals } \\
\text { - Participants will be informed about acupuncture } \\
\text { treatment in the study as follows: } \\
\text { "In this study, classic and nonclassic acupuncture } \\
\text { treatment for GERD will be used based on traditional } \\
\text { Korean medicine textbook and GERD- related } \\
\text { literature." }\end{array}$ \\
\hline $\begin{array}{l}\text { 5. Practitioner } \\
\text { background }\end{array}$ & $\begin{array}{l}\text { 5) Description of participating acupuncturists } \\
\text { (qualification or professional affiliation, years in } \\
\text { acupuncture practice, other relevant experience) } \\
\text { - Two Korean Medicine Doctors who have licenses } \\
\text { and at least } 5 \text { years of experience in gastrointestinal } \\
\text { disorders. They went through training and simulation } \\
\text { to ensure that they are able to provide identical EA } \\
\text { treatment on the ground of predefined protocol and } \\
\text { standard operating procedure. }\end{array}$ \\
\hline $\begin{array}{l}\text { 6. Control or } \\
\text { comparator } \\
\text { interventions }\end{array}$ & $\begin{array}{l}\text { 6a) Rationale for the control or comparator in the } \\
\text { context of the research question, with sources that } \\
\text { justify this choice } \\
\text { - A sham EA group using Park sham device with sham } \\
\text { needle will be adopted. }\end{array}$ \\
\hline & $\begin{array}{l}\text { 6b) Precise description of the control or comparator. } \\
\text { If sham acupuncture or any } \\
\text { other type of acupuncture-like control is used, } \\
\text { provide details as for Items } 1 \text { to } 3 \text { above. } \\
\text { - Participants in the sham EA group will receive } \\
\text { acupuncture treatment after } \\
\text { randomization twice weekly for } 6 \text { weeks. } \\
\text { - Sham EA group: nonpenetrating, nonacupoint, } \\
\text { nonelectric current } \\
\text { (1) Device: Park sham device with sham needle } \\
\text { (2) Acupoint: Eighteen acupoints. Each acupoint is } \\
2 \text { cm apart from the acupoints of EA group to the } \\
\text { medial side. (The optional points in accordance } \\
\text { with pattern identification will not be included) } \\
\text { (3) Duration of treatment sessions: } 20 \text { minutes }\end{array}$ \\
\hline
\end{tabular}

STRICTA STandards for Reporting Interventions in Clinical Trials of Acupuncture; $E A$ elctroacupuncture, GERD, gastroesophageal reflux disease, $S T$ stomach, $P C$ pericardium, $L /$ large intestine, $L R$ liver, $S P$ spleen, $G B$ gallbladder, CV conception vessel

\section{Primary outcome}

We set the "proportion of responders" (PR) [28, 29] as the primary outcome. PR is the proportion of participants who respond "yes" to more than half of the ARrelated questions during the treatment period [30]. An assessor will ask the participants the following question at each visit during the treatment period (twice a week for 6 weeks): "Have you had adequate relief of the pain or discomfort caused to you by GERD since the last visit?" We will define AR as "improvement of GERD symptoms by $\geq 20 \%$ since the last visit. 
Table 2 Study schedule for data collection

\begin{tabular}{|c|c|c|c|c|c|c|c|c|c|c|c|c|c|}
\hline Measures & V1 & V2 & V3 & V4 & V5 & V6 & V7 & V8 & V9 & V10 & V11 & V12 & V13 \\
\hline Sociodemographic data & $\checkmark$ & & & & & & & & & & & & \\
\hline GERD pattern identification tool & $\checkmark$ & & & & & & & & & & & & $\checkmark$ \\
\hline Collection and distribution of diary & $\checkmark$ & & $\checkmark$ & & $\checkmark$ & & $\checkmark$ & & $\checkmark$ & & $\checkmark$ & & $\checkmark$ \\
\hline Assessment of beliefs in the acupuncture effectiveness & $\checkmark$ & & & & & & & & & & & & \\
\hline Adequate relief (AR) & & $\checkmark$ & $\checkmark$ & $\checkmark$ & $\checkmark$ & $\checkmark$ & $\checkmark$ & $\checkmark$ & $\checkmark$ & $\checkmark$ & $\checkmark$ & $\checkmark$ & $\checkmark$ \\
\hline Nepean Dyspepsia Index - Korean (NDI-K) & $\checkmark$ & & & & & $\checkmark$ & & & & & & & $\checkmark$ \\
\hline Numeric rating scale (NRS) for GERD & $\checkmark$ & & & & & $\checkmark$ & & & & & & & $\checkmark$ \\
\hline Korean Gastrointestinal Symptom rating scale (KGSRS) & $\checkmark$ & & & & & $\checkmark$ & & & & & & & $\checkmark$ \\
\hline EuroQol-5 Dimension (EQ-5D) & $\checkmark$ & & & & & $\checkmark$ & & & & & & & $\checkmark$ \\
\hline Perceived Stress Scale-Korean (PSS) & $\checkmark$ & & & & & $\checkmark$ & & & & & & & $\checkmark$ \\
\hline Gut hormones (gastrin and motilin) & $\checkmark$ & & & & & & & & & & & & $\checkmark$ \\
\hline Blood collection for cytokine analysis & $\checkmark$ & & & & & & & & & & & & $\checkmark$ \\
\hline Qi stagnation and spleen Qi deficiency questionnaire & $\checkmark$ & & & & & & & & & & & & $\checkmark$ \\
\hline PGIC GERD symptom & & & & & & $\checkmark$ & & & & & & & $\checkmark$ \\
\hline \multicolumn{13}{|l|}{ Blinding Index (BI) and cCredibility test } & $\checkmark$ \\
\hline Adverse events investigation & & $\checkmark$ & $\checkmark$ & $\checkmark$ & $\checkmark$ & $\checkmark$ & $\checkmark$ & $\checkmark$ & $\checkmark$ & $\checkmark$ & $\checkmark$ & $\checkmark$ & $\checkmark$ \\
\hline Assessment of concomitant therapy and lifestyle modification & $\checkmark$ & $\checkmark$ & $\checkmark$ & $\checkmark$ & $\checkmark$ & $\checkmark$ & $\checkmark$ & $\checkmark$ & $\checkmark$ & $\checkmark$ & $\checkmark$ & $\checkmark$ & $\checkmark$ \\
\hline Evaluation of rescue medicines & & $\checkmark$ & $\checkmark$ & $\checkmark$ & $\checkmark$ & $\checkmark$ & $\checkmark$ & $\checkmark$ & $\checkmark$ & $\checkmark$ & $\checkmark$ & $\checkmark$ & $\checkmark$ \\
\hline
\end{tabular}

If participants report AR at more than half of all treatment sessions, they will be defined as responders, and we will compare the proportion of responders between the EA and sham EA groups.

\section{Secondary outcomes}

Nepean dyspepsia index - Korean version The Nepean dyspepsia index (NDI), a disease-specific instrument used to assess GERD symptoms [31], is a reliable and valid questionnaire [32, 33]. In this trial, the Korean version of NDI (NDI-K) will be used. It was validated in 2004 by Lee et al. [34] and contains 40 items comprising two sections: symptom-based questions (duration, severity, and degree of 15 separate symptoms), and those pertaining to the quality of life (QoL). The patient will complete the questionnaire at baseline, visit 6 , and visit 12 .

Gastrointestinal hormone (gastrin, motilin) GERD is related to weakness in the lower esophageal sphincter [35], and motilin [36] and gastrin [37, 38] have been reported to increase strength of the lower ESP (LESP). Motilin is an endogenous gastrointestinal hormone that increases upper GI tract motility, and it is considered an endogenous prokinetic hormone [39]. Gastrin is also a gastrointestinal polypeptide; it is thought to stimulate the lower esophageal sphincter by increasing the LESP [40]. However, controversy exists regarding whether EA increases gastrin and motilin levels [11, 13-16], so we will attempt to confirm the influence of EA on increases in motilin and gastrin in this trial. Specifically, a nurse will collect a 10-mL blood sample, in which the serum will be separated using a centrifuge. The sample will be analyzed using both a Human Motilin ELISA kit and a Human Gastrin ELISA kit (Mybiosource Inc., San Diego, California, USA). This will be carried out at baseline and visit 12 .

Inflammatory cytokines One report has claimed that cellular inflammatory processes are involved in the pathogenesis of GERD and its complications [17]. On this basis, we will evaluate serum IL-1, IL-6, IL-8, IL-4, and IL-10 (all inflammatory mediators) using the $\mathrm{BD}$ Facscalibur ${ }^{\text {rin }}$ (BD Bioscience, CA, USA) at baseline and visit 12 . IL-8 is expressed at high levels in the affected mucosae of patients with GERD [41-43]. IL-1, IL-6, IL-10, and IL-4 are also important mediators of the inflammatory responses related to GERD [17]. Through this analysis, we will clarify the influence of EA on the levels of cytokines, as well as the relation between these results and subjective symptoms.

EuroQol-5 Dimension The EuroQol-5 Dimension (EQ-5D) [44] will be used to evaluate the participants' QoL. The EQ-5D is a standardized preference-based questionnaire for measuring health-related QoL [30]; it consists of the EQ-5D index and the EQ-5D visual analog scale (VAS). The EQ-5D index is calculated on the basis of five dimensions: mobility, self-care, ability to undertake usual activities, pain, and anxiety/depression. The EQ-5D VAS measures the participant's current health status using a standard vertical $20 \mathrm{~cm}$ 
VAS [45]. The top of this line is labeled "best imaginable health state" and the bottom "worst imaginable health state." We will use a validated Korean version of EQ-5D [46] at baseline, visit 6, and visit 12 .

Korean gastrointestinal symptom rating scale The Korean gastrointestinal symptom rating scale (KGSRS) [47] will be used to evaluate the gastrointestinal discomfort of participants. This questionnaire consists of 16 items covering both upper and lower gastrointestinal symptoms. In this trial, we will evaluate changes in reflux, abdominal pain, and indigestion after treatment. The evaluation will be carried out at baseline, visit 6, and visit 12 .

Eleven-point numerical rating scale Participants will be asked to check the discomfort caused to them by GERD using an 11-point numerical rating scale. This will be performed at baseline, visit 6 , and visit 12 .

Perceived stress scale The perceived stress scale (PSS) was developed and validated by Cohen et al. in 1983 [48]. We will use the PSS translated into Korean and validated by Lee [49]. This questionnaire evaluates a participant's perceived stress during the last 1 month using a 5-point scale.

Blinding index and credibility assessment Blinding of participants will be assessed using a blinding index (BI) [50]. Participants will answer the following question for each acupuncture point: "What type of acupuncture do you think you received?" The answer will be selected from three choices: "classic electroacupuncture," "nonclassic electroacupuncture," or "don't know." In addition, the credibility of the treatment will be evaluated using the credibility assessment questionnaire. These evaluations will be conducted after EA treatment at visit 12 .

Pattern identification for gastroesophageal reflux disease The participants' pattern will be classified using pattern identification for gastroesophageal reflux disease (PIGERD) [21]. Participants will be assigned EA treatment on the basis of the result of the PIGERD. This identification will be performed at visit 1 and visit 12 .

The acupuncture beliefs scale We will assess the participant's belief in the efficacy of EA at visit 1 using the acupuncture beliefs scale [51], which consists of 36 items. Participants will respond on a 5-point Likert scale as follows: "strongly agree" (1 point), "agree" ( 2 points), "neutral" ( 3 points), "disagree" (4 points), and "strongly disagree" (5 points).

Patient global impression of change The EA will be globally assessed by the participants using patient global impression of change-a self-reported seven-point categorical scale that is used to evaluate overall improvement after EA treatment [52]. Participants will evaluate improvements in their symptoms between baseline and visit 6 , as well as between baseline and visit 12 , by choosing one of the following answers: (1) very much improved, (2) much improved, (3) minimally improved, (4) no change, (5) minimally worse, (6) much worse, or (7) very much worse.

Qi-stagnation questionnaire In traditional Korean medicine, the concept of qi stagnation is similar to that of stress in Western thinking [53]. The qi stagnation questionnaire is composed of 11 subjective items and one objective item; the items are used to diagnose qi stagnation. Using this questionnaire, we will evaluate the degree of qi stagnation in patients with GERD at visit 1 and visit 12 .

Spleen qi deficiency questionnaire The spleen qi deficiency questionnaire [54] will be used to diagnose whether the participant is in a state of spleen qi deficiency. This questionnaire consists of nine items concerning symptoms and two items regarding tongue and pulse diagnosis. The diagnosis will be performed at visit 1 and visit 12 by a KMD.

Evaluation of rescue medicine We will quantify the amount of rescue medicines taken between visits.

\section{Sample size calculation}

The required sample size was calculated on the basis of the difference in PR between the EA and sham EA groups. On the basis of previous research [55], we assumed the PR would be $92.8 \%$ in the treatment group and $57.1 \%$ in the control group. With a $5 \%$ false positive error $(\alpha=0.05$, two-sided) and $80 \%$ power $(\beta=0.2)$ the required sample size in each group was $n=21.9$. Assuming a $25 \%$ of dropout rate, the real-world sample size will be $n=30$ in each group, for a total of 60 participants.

\section{Statistical analysis}

An independent statistician blinded to group allocation will carry out the statistical analysis using SPSS $^{\text {mix }} 18.0$ (SPSS Inc., Chicago, Illinois, USA). All final data will be securely stored, and only the statistician will have access. We will perform both intention-to-treat (ITT) and per protocol (PP) analysis, with all analyses being based on the ITT principle. Any participant who receives EA more than nine times during the study period will be eligible for PP analysis.

For ITT analysis of secondary outcomes, we will use the "last observation carried forward" rule. In the case of the primary outcome, however, missing values will not be replaced, because of the characteristics of adequate relief (AR). We will only count actual responses regarding AR. 
When AR occurs in more than $50 \%$ of the treatment sessions in which a study subject participated, the participant will be classified as a responder. All data will be shown as the mean \pm standard deviation (SD) or as numbers and percentages. The statistical significance level will be set at 0.05 (two-sided), with $95 \%$ confidence intervals (CI). The analysis for safety will also follow the principles of ITT.

\section{Description of baseline characteristics and homogeneity of the two groups}

To describe the baseline characteristics of continuous data, the mean $\pm S D$, or range with minimum and maximum, will be used. In the case of dichotomous data, the frequency will be reported as a percentage. To evaluate the homogeneity of the baseline characteristics between the two groups, a two-sample $t$ test for continuous data and the $\chi^{2}$ test for dichotomous data will be carried out. If there is a significant difference between the two groups in terms of baseline characteristics, we will use analysis of covariance (ANCOVA) or logistic regression.

\section{Primary outcome}

The primary outcome-PR after 6 weeks of EA-will be compared between the groups using the $\chi^{2}$ or Fisher's exact test.

\section{Secondary outcomes}

The independent $t$ test will be used to evaluate changes in the NDI-K score after 6 weeks of treatment. If there is a covariate at baseline, an ANCOVA will be performed. To compare variables between the two groups, the twosample $\mathrm{t}$ test will be used for parametric analyses, and the Mann-Whitney U test, for nonparametric analyses.

A repeated measures analysis of variance or repeated measured ANCOVA will be used to analyze group-bytime interactions, as well as intergroup differences. The $\chi^{2}$ test or Fisher's exact test will be used to analyze nominal variables in each group as well as to compare AEs between groups.

\section{Data management}

A well-trained assessor will collect data during the study period, and a blinded data manager will enter the data from the case report form into an electronic database. All data will be crosschecked and accessible only to the data manager, who is independent from the sponsor and competing interests.

\section{Safety}

We will ask participants at each visit whether any $\mathrm{AE}$ has occurred or not. If any AEs occur, we will offer immediate and appropriate treatment to the participant and observe his/her progression through follow up. In addition, we will record the name, severity, duration, and cause of all AEs that occur during the period of study. Then, the investigator will assess causality between the $\mathrm{AE}$ and the intervention. If a correlation is noted, the investigator will take prompt and appropriate action. The causes of AEs during EA treatment will be reviewed by an independent monitor. If any serious $\mathrm{AE}$ occurs, we will report it to the Institutional Review Board (IRB) within 24 hours of recognition.

\section{Quality control}

A well-trained clinical research associate independent from the investigators will regularly moderate the quality of this trial. The monitoring will consist of checking (1) informed consent and case report forms, (2) participants' compliance with treatments, (3) the trial master file, (4) serious AEs, and (5) the data set.

\section{Ethical approval and registration}

We will carry out this trial according to the standards of the International Committee on Harmonization of Good Clinical Practice and the revised version of the Declaration of Helsinki. An initial version of this protocol was approved by Kyung Hee University Korean Medicine Hospital IRB (KOMCIRB-150217-HRBR-009) on March 27, 2015.

If there is change in the protocol, the investigator will report this change to the relevant parties, such as the IRB, and trial participants. In addition, the investigators will be educated in maintaining the confidentiality of the participants' personal information. This study is registered as a clinical trial at the Clinical Research Information Service (CRIS; KCT0001653; https://cris.nih.go.kr/cris/en/).

\section{Discussion}

The current study will be a randomized, patient-blinded, assessor-blinded, sham-controlled trial to evaluate the effect of EA on GERD symptoms, as well as to clarify the influence of EA on esophageal motility-related variables. To our knowledge, insufficient evidence exists regarding the efficacy of EA in patients with GERD. Among the few studies that do exist on the topic, not one has used the Park sham device as a control. This trial is expected to clarify whether EA provides adequate symptom relief and improves QoL and whether GI hormone secretion and inflammatory cytokines are more affected by EA than by sham EA.

The Park device is the most commonly used placebo in acupuncture research [56], and it has been used as a control in many acupuncture RCTs [23-25, 57]. The device consists of a needle (either penetrating or blunted) and an additional plastic tube, and it has been validated in a previous study [58]; the two needle types are visually indistinguishable and have specific modalities. Therefore, we will use the Park device as a placebo control.

In this study, participants will only be included if they suffer GERD symptoms despite having undergone standard 
treatments, such as PPIs or lifestyle modification, before enrollment. In addition, we will allow the participants in both groups to modify their lifestyles as a part of conventional care. For this reason, we do not believe that withholding PPI treatment will raise an ethical problem. The manner of lifestyle modification varies; we have chosen a protocol from the Korean clinical practice guidelines because we would like to reflect the real setting of Korean clinical practice. In addition, we will check whether the lifestyle modifications have been well maintained.

One report addressing functional gastrointestinal disorders (FGIDs) recommended that participants be classified as either responders or nonresponders in terms of the primary outcome [28], whereas another study showed that a responder analysis may be an important addition to clinical trial reporting [59]. Still another [30] used PR as a primary outcome for evaluating the effect of acupuncture in functional dyspepsia, which is one of the FGIDs. Hence, we will also use PR as a primary outcome in this trial.

We have set the duration of EA at 6 weeks in the current study to reflect recommendations regarding treatment trials involving FGIDs. This study, therefore, will be able to test for an adequate duration of EA as well as to reflect clinical practice.

We will also evaluate the levels of gastrin, motilin, and inflammatory cytokines such as IL-1, IL-4, IL-6, and IL-8; all these are objective outcomes related GERD. In this way, we will elucidate the mechanism of GERD symptom improvement, as well as the correlation between objective and subjective, symptomrelated outcomes.

This study has the following limitations. First, doctors will not be blinded to the group allocation because of the features of the Park device. Blinding implies keeping those who have an important role in the research, such as doctors who perform treatment, participants, outcome assessors, and data analysts, unaware of the treatment administered [60]. To overcome this limitation, the outcome assessor will be separated from the doctors, and the blinded assessor will measure and collect all outcome measures. Another limitation is that we will include participants without performing gastroscopy; rather, we will take a history from the participants. However, this may be regarded as pragmatic because it will more accurately reflect common clinical practice in Korean medicine.

This will be the first well-designed RCT using a validated device as a placebo control. The results will provide information about the efficacy and safety of EA as a treatment for GERD symptoms as well as evidence regarding the application of EA to treat GERD in real clinical practice.

\section{Trial status}

We will recruit participants from August 2015. Interim analyses are not planned, and the primary results will be published by 2016 .

\section{Additional file}

Additional file 1: SPIRIT 2013 Checklist. Completed SPIRIT checklist.

(PDF $57 \mathrm{~kb})$

\begin{abstract}
Abbreviations
GERD: gastroesophageal reflux disease; PPI: proton pump inhibitor;

EA: electroacupuncture; ESP: esophageal sphincter pressure; TLESRs: transient lower esophageal sphincter relaxations; RCTs: randomized controlled trials;

KMD: Korean Medicine Doctor; SOP: standard operating procedure;

STRICTA: Standards for Reporting Interventions in Clinical Trials of

Acupuncture; AR: adequate relief; PR: proportion of responders; NDI-

K: Nepean dyspepsia index —Korean version; EQ-5D: European Quality of

life-5 Dimension; PSS: perceived stress scale; ANCOVA: analysis of covariance;

AEs: adverse events; IRB: Institutional Review Board; FGIDs: functional

gastrointestinal disorders.
\end{abstract}

Competing interests

The authors declare that they have no competing interests.

\section{Authors' contribution}

GJH contributed to the conception and design, manuscript writing, critical revision, and final approval of the manuscript. JTL contributed to the conception and design, manuscript writing, critical revision, and final approval of the manuscript. HJL contributed to the critical revision and final approval of the manuscript. JHL contributed to the conception and design, manuscript writing, critical revision, financial support, and final approval of the manuscript. All authors read and approved the final manuscript before submission.

\section{Acknowledgements}

This study was supported by a grant of the Traditional Korean Medicine R\&D Project, Ministry of Health \& Welfare, Republic of Korea. (HI13C0700).

\section{Author details}

${ }^{1}$ Korean Medicine Clinical Trial Center, Kyung Hee University Korean Medicine Hospital, 23 Kyungheedae-ro, Dongdaemun-gu, Seoul 02447, Republic of Korea. ${ }^{2}$ Department of Gastroenterology, College of Korean Medicine, Kyung Hee University, 26 Kyungheedae-ro, Dongdaemun-gu, Seoul 02447, Republic of Korea. ${ }^{3}$ Department of Clinical Korean Medicine, Graduate School, Kyung Hee University, 26 Kyungheedae-ro, Dongdaemun-gu, Seoul 02447, Republic of Korea. ${ }^{4}$ Department of Sasang Constitutional Medicine, College of Korean Medicine, Kyung Hee University, 26 Kyungheedae-ro, Dongdaemun-gu, Seoul 02447, Republic of Korea.

Received: 23 November 2015 Accepted: 28 April 2016

Published online: 17 May 2016

\section{References}

1. Goh K-L. Gastroesophageal reflux disease in Asia: A historical perspective and present challenges. J Gastroenterol Hepatol. 2011;26 Suppl 1:2-10.

2. Ho KY, Cheung TK, Wong BC. Gastroesophageal reflux disease in Asian countries: disorder of nature or nurture? J Gastroenterol Hepatol. 2006;21:1362-5.

3. Ho KY. Gastroesophageal reflux disease in Asia: a condition in evolution. J Gastroenterol Hepatol. 2008;23:716-22.

4. Hayden CW, Bernstein CN, Hall RA, Vakil N, Garewal HS, Fass R. Usage of supplemental alternative medicine by community-based patients with gastroesophageal reflux disease (GERD). Dig Dis Sci. 2002;47:1-8.

5. Dickman R, Schiff E, Holland A, Wright C, Sarela SR, Han B, et al. Clinical trial: acupuncture vs. doubling the proton pump inhibitor dose in refractory heartburn. Aliment Pharmacol Ther. 2007;26:1333-44. 
6. El-Serag $H$, Becher A, Jones R. Systematic review: persistent reflux symptoms on proton pump inhibitor therapy in primary care and community studies. Aliment Pharmacol Ther. 2010;32:720-37.

7. Zhang C, Guo L, Guo X, Guo X, Li G. Clinical curative effect of electroacupuncture combined with zhizhukuanzhong capsules for treating gastroesophageal reflux disease. J Tradit Chin Med. 2012;32:364-71.

8. Qian LW, Lin YP. Effect of electroacupuncture at zusanli (ST36) point in regulating the pylorus peristaltic function. Zhongguo Zhong Xi Yi Jie He Za Zhi. 1993;13:336-9. 324.

9. Shuai X, Xie P, Liu J, Xiang Y, Li J, Lan Y. Different effects of electroacupuncture on esophageal motility and serum hormones in cats with esophagitis. Dis Esophagus Off J Int Soc Dis Esophagus ISDE. 2008;21:170-5.

10. Zhou $L$. The regulatory function of acupuncture of the stomach. Acupunct Res. 1986;11:274-9.

11. Wang C, Zhou D-F, Shuai X-W, Liu J-X, Xie P-Y. Effects and mechanisms of electroacupuncture at PC6 on frequency of transient lower esophageal sphincter relaxation in cats. World J Gastroenterol. 2007:13:4873-80.

12. Xing J, Chen JDZ. Alterations of gastrointestinal motility in obesity. Obes Res. 2004;12:1723-32.

13. Chang C-S, Chou J-W, Wu C, Chang Y-H, Ko C-W, Chen G-H. Atropineinduced gastric dysrhythmia is not normalized by electroacupuncture. Dig Dis Sci. 2002;47:2466-72.

14. Tougas G, Yuan LY, Radamaker JW, Chiverton SG, Hunt RH. Effect of acupuncture on gastric acid secretion in healthy male volunteers. Dig Dis Sci. 1992:37:1576-82

15. Niu W-X, He G-D, Liu H, Qin X-Y. Effects and probable mechanisms of electroacupuncture at the Zusanli point on upper gastrointestinal motility in rabbits. J Gastroenterol Hepatol. 2007;22:1683-9.

16. Lee CH, Kim D-K, Yook T-H, Sasaki M, Kitamura N. Effectiveness of electroacupuncture at Zusanli (ST36) on the immunohistochemical density of enteroendocrine cells related to gastrointestinal function. J Acupunct Meridian Stud. 2012;5:63-71.

17. Rieder F, Biancani P, Harnett K, Yerian L, Falk GW. Inflammatory mediators in gastroesophageal reflux disease: impact on esophageal motility, fibrosis, and carcinogenesis. Am J Physiol Gastrointest Liver Physiol. 2010;298:G571-81.

18. Jeong YJ, Lee $\mathrm{DH}$, Choi TH, Hwang TJ, Lee BH, Nah JC, et al. Clinical analysis of recurrence rate and symptom improvement in gastro-esophageal reflux disease patients. Korean J Gastroenterol. 2010;55:100-8.

19. Chiu C-T, Hsu C-M, Wang C-C, Chang J-J, Sung C-M, Lin C-J, et al. Randomised clinical trial: sodium alginate oral suspension is non-inferior to omeprazole in the treatment of patients with non-erosive gastroesophageal disease. Aliment Pharmacol Ther. 2013:38:1054-64.

20. MacPherson H, Altman DG, Hammerschlag R, Youping L, Taixiang W, White A, et al. STRICTA revision group: Revised STandards for Reporting Interventions in Clinical Trials of Acupuncture (STRICTA): extending the CONSORT statement. PLoS Med. 2010;7, e1000261.

21. Han G, Leem J, Lee N, Kim J, Park J, Lee J. Development of a standard tool for pattern identification of gastroesophageal reflux disease (GERD). J Korean Orient Intern Med. 2015:36:122-52.

22. Park J, White A, Lee H, Ernst E. Development of a new sham needle. Acupunct Med. 1999;17:110-2.

23. Smith P, Mosscrop D, Davies S, Sloan P, Al-Ani Z. The efficacy of acupuncture in the treatment of temporomandibular joint myofascial pain: a randomised controlled trial. J Dent. 2007;35:259-67.

24. Whale CA, MacLaran SJA, Whale Cl, Barnett M. Pilot study to assess the credibility of acupuncture in acute exacerbations of chronic obstructive pulmonary disease. Acupunct Med. 2009;27:13-5.

25. Chae $Y$, Lee $H$, Kim H, Sohn H, Park J-H, Park H-J. The neural substrates of verum acupuncture compared to non-penetrating placebo needle: an fMRI study. Neurosci Lett. 2009;450:80-4

26. Jung $H$, Hong $S$, Jo $Y$, Jeon $S$, Cho $Y$, Lee $K$, Lee J, Park H, Shin E, Lee S, Han S. The Korean Society of Neurogastroenterology and Motility: updated guidelines for gastroesophageal reflux disease. Korean J Gastroenterol. 2012; 2012(60):195-218

27. Kahrilas PJ, Shaheen NJ, Vaezi MF, American Gastroenterological Association Institute, Clinical Practice and Quality Management Committee. American Gastroenterological Association Institute technical review on the management of gastroesophageal reflux disease. Gastroenterology. 2008;135:1392-413. 1413.e1-5.

28. Design of Treatment Trials Committee, Irvine EJ, Whitehead WE, Chey WD, Matsueda K, Shaw M, et al. Design of treatment trials for functional gastrointestinal disorders. Gastroenterology. 2006;130:1538.
29. Talley NJ, Van Zanten SV, Saez LR, Dukes G, Perschy T, Heath M, et al. A dose-ranging, placebo-controlled, randomized trial of alosetron in patients with functional dyspepsia. Aliment Pharmacol Ther. 2001;15:525-37.

30. Han G, Ko S-J, Park J-W, Kim J, Yeo I, Lee H, et al. Acupuncture for functional dyspepsia: study protocol for a two-center, randomized controlled trial. Trials. 2014;15:89.

31. Bolier EA, Kessing BF, Smout AJ, Bredenoord AJ. Systematic review: questionnaires for assessment of gastroesophageal reflux disease. Dis Esophagus. 2015;28:105-20.

32. Talley NJ, Haque M, Wyeth JW, Stace NH, Tytgat GN, Stanghellini V, et al. Development of a new dyspepsia impact scale: the Nepean Dyspepsia Index. Aliment Pharmacol Ther. 1999;13:225-35.

33. Talley NJ, Verlinden M, Jones M. Quality of life in functional dyspepsia: responsiveness of the Nepean Dyspepsia Index and development of a new 10-item short form. Aliment Pharmacol Ther. 2001:15:207-16.

34. Lee S, Choi S, Cho Y, Choi M. Validation of the Nepean dyspepsia index Korean version. Kor J Neurogastroenterol Mot. 2003:9(suppl):48S.

35. Stein HJ, Barlow AP, DeMeester TR, Hinder RA. Complications of gastroesophageal reflux disease. Role of the lower esophageal sphincter, esophageal acid and acid/alkaline exposure, and duodenogastric reflux. Ann Surg. 1992;216:35-43.

36. Lux G, Rösch W, Domschke S, Domschke W, Wünsch E, Jaeger E, et al. Intravenous 13-Nle-motilin increases the human lower esophageal sphincter pressure. Scand J Gastroenterol Suppl. 1976;39:75-9.

37. Castell DO, Harris LD. Hormonal control of gastroesophageal-sphincter strength. N Engl J Med. 1970;282:886-9.

38. Lepsien $\mathrm{G}$, Dietrich K. Peptone stimulation of the lower esophageal sphincter in patients with reflux disease. Z Für Gastroenterol. 1988:26:209-16.

39. Korimilli A, Parkman HP. Effect of atilmotin, a motilin receptor agonist, on esophageal, lower esophageal sphincter, and gastric pressures. Dig Dis Sci. 2010;55:300-6.

40. Straathof JW, Lamers CB, Masclee AA. Effect of gastrin-17 on lower esophageal sphincter characteristics in man. Dig Dis Sci. 1997;42:2547-51.

41. Fitzgerald RC, Onwuegbusi BA, Bajaj-Elliott M, Saeed IT, Burnham WR, Farthing MJG. Diversity in the oesophageal phenotypic response to gastrooesophageal reflux: immunological determinants. Gut. 2002;50:451-9.

42. Isomoto $H$, Saenko VA, Kanazawa $Y$, Nishi $Y$, Ohtsuru A, Inoue $K$, et al. Enhanced expression of interleukin-8 and activation of nuclear factor kappa$B$ in endoscopy-negative gastroesophageal reflux disease. Am J Gastroenterol. 2004;99:589-97.

43. Yoshida N, Uchiyama K, Kuroda M, Sakuma K, Kokura S, Ichikawa H, et al. Interleukin-8 expression in the esophageal mucosa of patients with gastroesophageal reflux disease. Scand J Gastroenterol. 2004;39:816-22.

44. Mahadeva S, Wee H-L, Goh K-L, Thumboo J. The EQ-5D (Eurogol) is a valid generic instrument for measuring quality of life in patients with dyspepsia. BMC Gastroenterol. 2009:9:20.

45. Kind P. The EuroQoL instrument: an index of health-related quality of life. In: Quality of life and pharmacoeconomics in clinical trials. Philadelphia: Lippincott-Raven; 1996. p. 191-201

46. Kang E, Park H, Kim N, Jo MW, Shin $\mathrm{H}$. A valuation of health status using EQ-5D. Korean J Health Econ Policy. 2006;12:19-43.

47. Kwon $\mathrm{S}$, Jung $\mathrm{H}$, Hong J, Park $\mathrm{H}$. Diagnostic validity of the Korean Gastrointestinal Symptom Rating Scale (KGSRS) in the assessment of gastroesophageal reflux disease. Ewha Med J. 2008:31:73-80.

48. Cohen S, Kamarck T, Mermelstein R. A global measure of perceived stress. J Health Soc Behav. 1983:24:385-96.

49. Lee E-H, Chung BY, Suh C-H, Jung J-Y. Korean versions of the Perceived Stress Scale (PSS-14, 10 and 4): psychometric evaluation in patients with chronic disease. Scand J Caring Sci. 2015:29:183-92.

50. Bang $H, \mathrm{Ni} L$, Davis CE. Assessment of blinding in clinical trials. Control Clin Trials. 2004:25:143-56.

51. Dennehy EB, Webb A, Suppes T. Assessment of beliefs in the effectiveness of acupuncture for treatment of psychiatric symptoms. J Altern Complement Med N Y N. 2002;8:421-5

52. Farrar JT, Young JP, LaMoreaux L, Werth $J \mathrm{~L}$, Poole RM. Clinical importance of changes in chronic pain intensity measured on an 11-point numerical pain rating scale. Pain. 2001:94:149-58.

53. Han G, Seon J, Son J, Oh S, Park Y, Jeong Y, et al. Correlation between xerostomia, stress, and Qi movement stagnation in halitosis patients. Korean Orient Intern Med. 2010;31:488-99. 
54. Oh HW. Study on the development of a standard instrument of diagnosis and assessment for spleen Qi deficiency pattern. J Korean Med Sci. 2014;35:157-70.

55. Yu D. Lansoprazole and mosapride with acupuncture treatment for 56 patients with non-erosive gastroesophageal reflux disease. Chinese Community Doctors. 2011;15:177-8.

56. Zhu D, Gao Y, Chang J, Kong J. Placebo acupuncture devices: considerations for acupuncture research. Evid-Based Complement Altern Med ECAM. 2013;2013:628907.

57. Pastore LM, Williams CD, Jenkins J, Patrie JT. True and sham acupuncture produced similar frequency of ovulation and improved LH to FSH ratios in women with polycystic ovary syndrome. J Clin Endocrinol Metab. 2011;96:3143-50.

58. Park J, White A, Stevinson C, Ernst E, James M. Validating a new nonpenetrating sham acupuncture device: two randomised controlled trials. Acupunct Med J Br Med Acupunct Soc. 2002;20:168-74.

59. Moore RA, Moore OA, Derry S, McQuay HJ. Numbers needed to treat calculated from responder rates give a better indication of efficacy in osteoarthritis trials than mean pain scores. Arthritis Res Ther. 2008;10:R39.

60. Schulz KF, Chalmers I, Altman DG. The landscape and lexicon of blinding in randomized trials. Ann Intern Med. 2002:136:254-9.

\section{Submit your next manuscript to BioMed Central and we will help you at every step:}

- We accept pre-submission inquiries

- Our selector tool helps you to find the most relevant journal

- We provide round the clock customer support

- Convenient online submission

- Thorough peer review

- Inclusion in PubMed and all major indexing services

- Maximum visibility for your research

Submit your manuscript at www.biomedcentral.com/submit 